Neurosurg Focus 14 (3):Introduction, 2003, Click here to return to Table of Contents

\title{
Cerebral Revascularization
}

\author{
Eric S. Nussbaum, M.D. \\ HealthEast Neurovascular Institute, St. Joseph's Hospital, St. Paul; and Fairview Medical Center, \\ Minneapolis, Minnesota
}

\begin{abstract}
Despite decades of critical analysis, the precise indications for cerebral revascularization remain poorly defined. It is clear that some patients undergoing therapeutic carotid artery (CA) sacrifice for the management of unclippable intracranial aneurysms or skull base neoplasms will require revascularization to prevent or reduce the risk of cerebral infarction.,11 Nevertheless, the decisions of when to perform this procedure and what type of bypass to undertake in these cases remain debated. The question of whether revascularization should be conducted in cases involving ischemic disease is even more controversial. ${ }^{1-3,5,8}$
\end{abstract}

This issue of Neurosurgical Focus is devoted to the topic of cerebral revascularization, assembling a collection of articles from noted experts in this field. The papers address many of the challenging and important issues yet to be defined in this controversial area.

In the lead article, Friedman and Piepgras review their extensive 15-year Mayo Clinic experience with saphenous vein grafts used for cerebral bypass. The trends that they identify regarding the decreasing number of such procedures are important both in terms of patient management and implications for future neurosurgical training. The reader should also note well the mention of several aneurysms requiring treatment with an intraoperative bypass that had not been planned preoperatively. As the incidence of surgically treated aneurysms decreases because of improving endovascular techniques, our facility with revascularization will likely continue to diminish. The concentration of these complex cases in centers of excellence where "unanticipated" revascularization can be performed when appropriate will become increasingly important if a meaningful level of technical competence with these demanding procedures is to be maintained.

In a pair of excellent review articles, Liu and colleagues describe a variety of strategies for the establishment of high-flow interpositional grafts and also describe the various conduits available for cerebral revascularization. Their discussion of the advantages and disadvantages of each approach will be of interest to anyone performing revascularization procedures and nicely complements the experience described by Friedman and Piepgras. Following these reviews, O'Shaughnessy and coworkers share with us an important description of their management philosophy for giant anterior circulation aneurysms. In this excellent article the authors elegantly illustrate a management strategy that has proven successful in the hands of one of the foremost cerebrovascular neurosurgeons in practice today. The thoughtful approach, which has clearly been refined repeatedly based on a careful analysis of practical experience, deserves detailed study.

Shifting gears to address the issue of preoperative assessment prior to CA sacrifice in patients with advanced head and neck cancer, Dare and associates describe their paradigm for balloon test occlusion (BTO) with hypotensive challenge. The question of which patients undergoing CA sacrifice will require revascularization remains a matter of debate, and at the present no single test or combination of studies can absolutely predict that a patient will tolerate CA sacrifice. ${ }^{7}$ This article, presented by the experienced group at Buffalo, highlights this point as one of their patients suffered a fatal ischemic insult despite passing the BTO. Continuing on the topic of CA occlusion in the setting of head and neck cancer, Feiz-Erfan and colleagues report the Barrow Neurological Institute experience involving bypass followed by radical excision of aggressive squamous cell carcinoma. As chemotherapy regimens and radiotherapy techniques improve, the neurosurgeon may be called upon to treat an increasing number of patients in whom more advanced neoplastic disease processes involve the CA., ${ }^{9,10}$ This paper nicely highlights the difficulties associated with managing this complicated subgroup of patients.

Turning to the issue of revascularization for ischemic disease, Melgar and Weinand describe their unusual experience in performing revascularization of the external carotid system in the treatment of postural cerebral ische- 
mia in patients with bilateral internal CA occlusion. Next, Tummala, et al., describe a large contemporary series of superficial temporal artery-middle cerebral artery anastomoses for occlusive cerebrovascular disease in cases treated at a center that has continued to champion the procedure in the management of patients refractory to maximal medical therapy. The final article represents an important contribution from Grubb and coworkers detailing the Carotid Occlusion Surgery Study project, a newly organized randomized trial examining the efficacy of extracranialintracranial bypass in the setting of unilateral symptomatic CA occlusion. ${ }^{6}$ Ultimately, it will likely be through this type of careful analysis that we may finally answer the question of when revascularization for ischemic disease should be performed.

In summary, the purpose of this issue of Neurosurgical Focus is to present the state-of-the-art thinking in the controversial field of cerebral revascularization. The articles, which touch on many aspects of this topic, underscore several important points that deserve to be emphasized.

1) A thoughtful and carefully studied approach is essential when treating patients in whom revascularization may be required for complex intracranial aneurysm, skull base tumor, or occlusive cerebrovascular disease. Because we lack hard scientific data to guide us in the management of these issues, we must rely on the carefully documented experience of individual surgeons who have dedicated their efforts to the management of these patients.

2) Cerebral bypass may unexpectedly be required intraoperatively, even by experienced surgeons; therefore, it is appropriate for neurosurgeons managing these complex disease processes to be facile with the various methods of cerebral revascularization.

3) With the increasing role of endovascular therapy in the treatment of intracranial aneurysms and occlusive cerebrovascular disease, the trend away from open microsurgery will continue, and fewer neurosurgeons will likely be competent to treat these problems. Therefore, referral of such complex patients to centers of excellence will be critical in the future.

4) It is the responsibility of those neurosurgeons who continue to treat these problems to attempt to refine our understanding of when to perform cerebral bypass and what type of bypass to perform when surgical revascularization is indicated.
Finally, I would like to acknowledge the excellent and timely efforts of Drs. Felipe Albuquerque and Jonathon Friedman who served as manuscript reviewers. I would also like to thank Susan Lanterman for her expert assistance with the preparation of this issue of Neurosurgical Focus, and I would remind the readership that we hope to create an ongoing dialogue on this topic. Articles addressing any aspect of cerebral revascularization may still be forwarded to Neurosurgical Focus for review and potential future publication.

\section{References}

1. Ausman JI, Diaz FG: Critique of the Extracranial-Intracranial bypass study. Surg Neurol 26:218-221, 1986

2. Awad IA, Spetzler RF: Extracranial-intracranial bypass: A critical analysis in light of the International Cooperative Study. Neurosurgery 19:655-664, 1986

3. Day AL, Rhoton AL, Little JR: The extracranial-intracranial bypass study. Surg Neurol 26:222-226, 1986

4. Drake CG, Peerless SJ, Ferguson GG: Hunterian proximal arterial occlusion for giant aneurysms of the carotid circulation. J Neurosurg 81:656-665, 1994

5. The EC/IC Bypass Study Group: Failure of extracranial-intracranial arterial bypass to reduce the risk of ischemic stroke: Results of an international randomized trial. N Engl J Med 313:1191-1200, 1985

6. Grubb RL Jr, Derdeyn CP, Fritsch SM, et al: Importance of hemodynamic factors in the prognosis of symptomatic carotid occlusion. JAMA 280:1055-1060, 1998

7. McIvor NP, Willinsky RA, TerBrugge KG, et al: Validity of test occlusion studies prior to internal carotid artery sacrifice. Head Neck 16:11-16, 1994

8. Nussbaum ES, Erickson DL: Extracranial-intracranial bypass for ischemic cerebrovascular disease refractory to maximal medical therapy. Neurosurgery 46:37-43, 2000

9. Nussbaum ES, Levine SC, Hamlar D, et al: Carotid stenting and "extarterectomy" in the management of head and neck cancer involving the internal carotid artery: Technical case report. Neurosurgery 47:981-984, 2000

10. Sessa CN, Morasch MD, Berguer R, et al: Carotid resection and replacement with autogenous arterial graft during operation for neck malignancy. Ann Vasc Surg 12:229-235, 1998

11. Spetzler RF, Shuster H, Roski R: Elective extracranial-intracranial arterial bypass in the treatment of inoperable giant carotid artery aneurysms. J Neurosurg 53:22-27, 1980 